\title{
Landmark-Constrained Elastic Shape Analysis of Planar Curves
}

\author{
Justin Strait, Sebastian Kurtek, Emily Bartha, Steven MacEachern \\ Department of Statistics, The Ohio State University
}

April 6, 2017

\begin{abstract}
Various approaches to statistical shape analysis exist in current literature. They mainly differ in the representations, metrics and/or methods for alignment of shapes. One such approach is based on landmarks, i.e., mathematically or structurally meaningful points, which ignores the remaining outline information. Elastic shape analysis, a more recent approach, attempts to fix this by using a special functional representation of the parametrically-defined outline in order to perform shape registration, and subsequent statistical analyses. However, the lack of landmark identification can lead to unnatural alignment, particularly in biological and medical applications, where certain features are crucial to shape structure, comparison, and modeling. The main contribution of this work is the definition of a joint landmark-constrained elastic statistical shape analysis framework. We treat landmark points as constraints in the full shape analysis process. Thus, we inherit benefits of both methods: the landmarks help disambiguate shape alignment when the fully automatic elastic shape analysis framework produces unsatisfactory solutions. We provide standard statistical tools on the landmark-constrained shape space including mean and covariance calculation, classification, clustering, and tangent principal component analysis (PCA). We demonstrate the benefits of the proposed framework on complex shapes from the MPEG-7 dataset and two real data examples: mice T2 vertebrae and Hawaiian Drosophila fly wings.
\end{abstract}

Keywords: Elastic metric, Geodesics, Statistics on shape spaces, Karcher mean, Tangent principal component analysis 


\section{Introduction}

An important physical characteristic of all objects observed in images is their shape. Most would define shape as the appearance of the outline or boundary of a particular object; it is a property that remains unchanged under rotation, translation, or scaling. This was the definition introduced by Kendall (1984) in his seminal paper. Statistical shape analysis is concerned with representing objects using their shapes, and developing mathematical and statistical tools for their analysis. It is important to note that standard statistical procedures often do not apply to shapes due to the nonlinearity and quotient structure of shape spaces. Thus, novel ideas are needed to study shape comparisons, impose statistical shape models, and develop inferential procedures on shape spaces. Since imaging data is ubiquitous in today's society, statistical shape analysis proves to be a very important and useful tool for applications in various areas including medical imaging, image processing and recognition, graphics, biology, and many other fields of study.

Due to the complexity of representing shapes mathematically, many different methods for shape analysis have been developed. One of the early tools used for statistical shape analysis involves the notion of landmarks: a collection of labeled points that are matched across a population of shapes, which represent important features of the global shapes of the objects under study. The importance could be spurred by extreme curvature of the outline or by a meaningful (semantic) interpretation of the objects' features. For example, in medical imaging, outlines of organs are often annotated with points of interest by a radiologist or doctor. In biometrics, a landmark may be placed at the nose, lips and eyes when looking at a facial side profile. The use of landmarks requires points on different object outlines to be identified in correspondence with each other. This removes the need for finding point correspondences across objects. Furthermore, landmark-based 
shape analysis can reduce the problem at hand to multivariate data analysis where existing statistical tools can be used with minor adjustments. Kendall (1984) is one of the pioneers of landmark analysis, and many others (including Dryden and Mardia (1998) and Small (1996)) have extended these concepts and developed statistical techniques on landmark shape spaces (Bookstein, 1986; Dryden and Mardia, 1992). However, representing an outline by a finite set of landmark points has its limitations, including:

1. Potentially limited knowledge about where landmarks are located on the outline.

2. The appropriate number of landmarks to select; shapes with more details may require more landmarks.

3. The ability to identify landmarks quickly for a large amount of shape data.

These drawbacks have been identified in multiple papers that have attempted to move beyond landmark-based shape analysis. One such improvement is to base shape representations not on a finite set of landmarks, but rather on the full curve defined by the boundary of the object. Such objects reside in infinite-dimensional spaces and thus require mathematical tools from functional data analysis. How the shape analysis of curves is performed has been subject to many different treatments. The main challenge arises from an additional property needed in the shape definition: invariance to re-parameterizations of the curves. A re-parameterization of a curve using an appropriate function is akin to changing the speed at which the curve is traversed (these ideas are defined in a mathematically precise manner in later sections). Given two curves, their re-parameterizations change the correspondence of geometric features. Many methods have been developed to deal with this issue. Some papers solve this problem by normalizing the parameterization of all curves to arc-length (Zahn and Roskies, 1972; Klassen et al., 2004). Unfortunately, as noted in several 
places (including in Figure 6 of Srivastava et al. (2011)), arc-length parameterizations tend to be suboptimal in many scientific problems of interest, as they do not match features between shapes well. A new direction considered searching for optimal parameterizations (correspondences) in a pairwise manner using elastic metrics (Younes, 1998; Joshi et al., 2007; Srivastava et al., 2011; Kurtek et al., 2012). There are two main advantages of elastic shape analysis frameworks over competitors: (1) rather than normalizing, optimal parameterizations are found to match geometric features across shapes, and (2) the distance between shapes is measured via elastic deformations, i.e., the amount of stretching and bending needed to deform one shape into another. The work of Srivastava et al. (2011), based on a special representation of curves called the square-root velocity function (SRVF), is the only method that applies to open and closed Euclidean curves in $\mathbb{R}^{n}$. This, along with the two previously mentioned advantages, makes it appealing for practical use.

The methodologies behind landmark shape analysis and elastic shape analysis appear very different. However, in many application areas there is a clear need to unify these two types of shape analysis techniques. In this paper, we consider the following natural question: With the ability to treat the outline of a shape directly as a function in an elastic framework, what additional information could knowledge of landmark points provide in shape comparisons and statistics? For instance, medical images contain full outlines of structures of interest, but also relevant anatomical landmarks detected automatically or provided by a domain expert. Thus, a reasonable approach to statistical shape analysis of anatomical structures would be to unify their representation by jointly analyzing the outline and landmark points.

As an example explored in later sections, consider the mouse vertebra in Figure 1. This dataset was originally analyzed under the landmark shape analysis approach in Dryden and Mardia (1992). In the left panel of this figure, we show the landmark representation of a mouse vertebra, which 


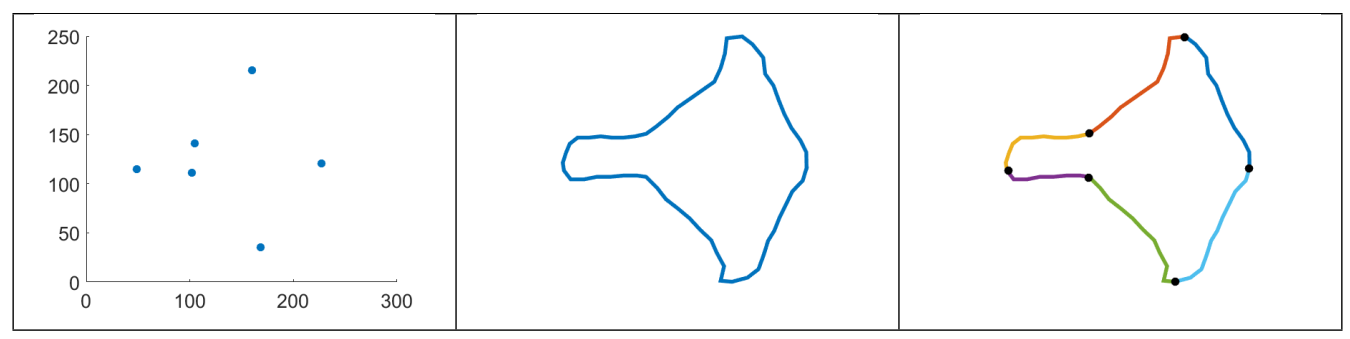

Figure 1: Example of a mouse vertebra represented via landmarks (left), full outline (center), and outline with landmarks superimposed (right).

is a crude approximation for the overall shape compared to the outline displayed in the middle panel. Therefore, it is more appealing to consider the entire outline to represent the data. But, we cannot forget that the provided landmarks have important meaning that is related to the anatomical structure of the vertebra. Rather than ignoring this information completely, as one would do under the elastic shape analysis framework, it seems more reasonable to incorporate these points into all shape analysis tasks. This results in hard landmark shape constraints, which play an important role in shape comparisons and models. This is the main goal of the current paper: to unify landmark and elastic shape analysis in a meaningful way.

The problem of incorporating landmark information in functional shape representations has been previously studied in the context of different shape frameworks. For example, Joshi and Miller (2000) described the joint problem under the large deformation diffeomorphism metric mapping (LDDMM) method. Wang et al. (2003) combined landmarks with topology-preserving shape deformations while Soatto and Yezzi (2002) solved the joint registration and segmentation problem for image analysis. However, these methods vary vastly in their shape representations from the method proposed in this paper. The only previous method that considered elastic shape analysis in the presence of landmarks did not address the issue of hard constraints (Liu et al., 2010). Instead, it incorporated landmarks into the curve representation by annotating the original outlines 
with functions derived from the landmark points. The main shortcoming of this approach is that one must choose a weight for the landmark information, which is a difficult task in practice. If this weight is small, the landmarks do not play a big role in the statistical analysis; when the weight is chosen to be very large, the landmark information dominates and the geometric information of the object outline is largely ignored. Another work considered the case of hard landmark constraints, but suffered from several theoretical and practical shortcomings (McCane, 2012). One theoretical shortcoming involved the pinching effect, which is discussed from the functional data perspective in Section 3.3 of Marron et al. (2015). This resulted in some additional practical issues where local, rather than global, rotations were used as the basis for finding optimal curve re-parameterizations during the shape analysis.

The rest of this paper is organized as follows. In Section 2, we describe the proposed landmarkconstrained elastic shape analysis framework for planar curves. The implementation of the method is discussed in Section 3. Section 4 provides a detailed description of three practical applications. We compare the proposed landmark-constrained elastic shape analysis to the unconstrained elastic method (as well as the landmark method in some cases). Finally, we provide a brief summary in Section 5 with a short discussion of directions for future work.

\section{Mathematical Framework}

In this section, we describe the proposed framework for statistical shape analysis of curves in the presence of hard landmark constraints. Note that this work builds on the elastic shape analysis framework of Srivastava et al. (2011). 


\subsection{Landmark-Constrained Elastic Shape Analysis of Curves}

Let $\beta:[0,1] \rightarrow \mathbb{R}^{2}$ be an absolutely continuous, open curve representing the outline of a planar object (for closed curves, the domain is represented by $\mathbb{S}^{1}$ ). While in this paper we focus on planar curves, the proposed method is easily modified to apply to open and closed curves in $\mathbb{R}^{n}$. As a first step, we seek a metric that is invariant to all shape preserving transformations of $\beta$. The simplest approach is to use the standard $\mathbb{L}^{2}$ metric for vector-valued functions to compare two curves $\beta_{1}$ and $\beta_{2}:\left\|\beta_{1}-\beta_{2}\right\|=\sqrt{\int_{0}^{1}\left|\beta_{1}(t)-\beta_{2}(t)\right|^{2} d t}$, where $|\cdot|$ is the Euclidean norm in $\mathbb{R}^{2}$. Unfortunately, while convenient, this metric lacks a key property for parameterization invariance. We explain this issue in more detail next. Let $\Gamma=\{\gamma:[0,1] \rightarrow[0,1] \mid \gamma(0)=0, \gamma(1)=1,0<\dot{\gamma}<\infty\}$ denote the re-parameterization group, where $\dot{\gamma}$ denotes the derivative of $\gamma$. Note that at this stage, $\Gamma$ is an unconstrained re-parameterization group. This group acts on the space of curves by composition, i.e., for a curve $\beta$ and a re-parameterization $\gamma$, the re-parameterized curve is given by $\beta \circ \gamma$. It is easy to see that the action of $\Gamma$ on the space of curves is not by isometries under the $\mathbb{L}^{2}$ metric: $\left\|\beta_{1}-\beta_{2}\right\| \neq\left\|\beta_{1} \circ \gamma-\beta_{2} \circ \gamma\right\|$. This prevents the $\mathbb{L}^{2}$ metric from being invariant to the action of $\Gamma$. Thus, one must turn to other ideas to establish invariance to re-parameterizations.

One way to fix the above-described issue is to establish a different representation of curves. The square-root velocity function (SRVF) is a commonly used representation, and was defined by Srivastava et al. (2011) as $q(t)=\frac{\dot{\beta}(t)}{\sqrt{|\dot{\beta}(t)|}}$. There are several benefits associated with this representation in the context of shape analysis:

1. The direction of the tangent vector to $\beta$ at any point along its parameterization, $t$, can be described by the unit $q$ vector at $t: \frac{\dot{\beta}(t)}{|\dot{\beta}(t)|}=\frac{q(t)}{|q(t)|}$. Changes to this direction vector result in bending-type deformations of curves. 
2. The instantaneous speed of $\beta$ at $t$ is $|\dot{\beta}(t)|=|q(t)|^{2}$. Changes in the instantaneous speed correspond to stretching-type deformations of curves.

3. The SRVF is automatically invariant to translations, since it only involves $\dot{\beta}$.

4. The mapping between $\beta$ and $(q, \beta(0))$ is a bijection where $\beta$ can be reconstructed using $\beta(t)=\beta(0)+\int_{0}^{t} q(s)|q(s)| d s$.

Scale invariance in this framework is achieved by re-scaling all curves to unit length $\int_{0}^{1}|\dot{\beta}(t)| d t=$ $\int_{0}^{1}|q(t)|^{2} d t=1$. The resulting SRVFs lie on the unit Hilbert sphere, which forms the pre-shape space: $\mathscr{C}=\left\{q:\left.[0,1] \rightarrow \mathbb{R}^{2}\left|\int_{0}^{1}\right| q(t)\right|^{2} d t=1\right\}$ (in the case of closed curves, there is an additional closure condition). We refer to $\mathscr{C}$ as the pre-shape space because while invariance to translation and scaling has been obtained, we have not yet dealt with rotation and re-parameterization. To achieve invariance to those transformations, we remove these two groups from the representation space algebraically through quotients.

At this stage, it is important to introduce the landmark constraints into the representation. While these constraints do not play a role in the definition of the SRVF and the scale normalization, they are involved in subsequent rotation and re-paremeterization steps. Thus, suppose that in addition to the full outline $\beta$, we are given $k$ discrete landmark points marked on $\beta,\left\{\beta\left(t_{1}\right), \ldots, \beta\left(t_{k}\right)\right\} \in \mathbb{R}^{2}$. Then, let $S O(2)$ be the standard representation space of all $2 \times 2$ rotation matrices. To account for hard landmark constraints, we must define a subgroup of $\Gamma$ whose elements respect landmark locations. In other words, the re-parameterization vector fields, which change the speed of traversal along the curve, must vanish at the landmark locations along the parameterization domain. Define $\Gamma_{0}=\left\{\gamma:[0,1] \rightarrow[0,1] \mid \gamma(0)=0, \gamma(1)=1,0<\dot{\gamma}<\infty, \gamma\left(t_{i}\right)=t_{i}, i=1, \ldots, k\right\}$ as the landmarkconstrained re-parameterization group. One can show that the actions of $S O(2)$ and $\Gamma_{0}$ commute. 
Furthermore, applying two such elements $O \in S O(2)$ and $\gamma \in \Gamma_{0}$ to a curve $\beta$ yields the transformed curve $O(\beta \circ \gamma)$; the SRVF of this transformed curve is given by $O(q \circ \gamma) \sqrt{\gamma}$. As a result, the landmark-constrained shape space $\mathscr{S}$ is defined by the set of equivalence classes $[q]=\{O(q \circ$ $\left.\gamma) \sqrt{\dot{\gamma}} \mid O \in S O(2), \gamma \in \Gamma_{0}\right\}$. Algebraically, $\mathscr{S}=\mathscr{C} /\left(\operatorname{SO}(2) \times \Gamma_{0}\right)$. The equivalence classes $[q]$ represent the landmark-constrained shapes uniquely, because any two shapes within a rotation and a constrained re-parameterization of each other are equivalent in the shape space. Therefore, by looking at the objects belonging to $\mathscr{S}$, the desired invariances to translation, scaling, rotation, and landmark-constrained re-parameterization have been achieved.

\subsection{Shape Comparisons via Landmark-Constrained Geodesics}

Finding an appropriate metric on $\mathscr{S}$ is the next task and can be addressed in a similar fashion to the unconstrained elastic framework. Given two shapes, the basic notion is to consider how one shape deforms into the other through the application of bending and stretching transformations; if this deformation process forms a longer path in $\mathscr{S}$, we expect the distance between the shapes to be larger. An important property of the elastic metric is that under the SRVF representation, this metric is equivalent to the standard $\mathbb{L}^{2}$ metric (Srivastava et al., 2011). Furthermore, it is easy to show that the $\mathbb{L}^{2}$ metric on $\mathscr{C}$ satisfies the isometry property for both $S O(2)$ and $\Gamma_{0},\left\|q_{1}-q_{2}\right\|=$ $\left\|O\left(q_{1} \circ \gamma\right) \sqrt{\dot{\gamma}}-O\left(q_{2} \circ \gamma\right) \sqrt{\dot{\gamma}}\right\|$, thus eventually allowing rotation and landmark-constrained reparameterization invariant shape comparisons. We define the distance between two SRVFs on $\mathscr{C}$ to be the length of the shortest path connecting them (a locally shortest geodesic). Since the pre-shape space $\mathscr{C}$ is the Hilbert sphere, we can use standard tools from the known differential geometry of this space. In particular, the geodesic path between two SRVFs $q_{1}, q_{2} \in \mathscr{C}$ is given by the great 
circle $\alpha_{q_{1}, q_{2}}(\tau)=\frac{1}{\sin (\theta)}\left(\sin ((1-\tau) \theta) q_{1}+\sin (\tau \theta) q_{2}\right), \tau \in[0,1]$, where $\theta=\cos ^{-1}\left(\left\langle q_{1}, q_{2}\right\rangle\right)$ is the length of this path or the geodesic distance between the two SRVFs, and $\langle\cdot, \cdot\rangle$ is the $\mathbb{L}^{2}$ inner product.

The rotation and landmark-constrained re-parameterization groups act on $\mathscr{C}$ by isometries, a property that allows the $\mathbb{L}^{2}$ metric to descend from the pre-shape space to the shape space (quotient space under the action of the product group). To obtain a geodesic on $\mathscr{S}$ (and thus have a distance between the equivalence classes $\left[q_{1}\right]$ and $\left[q_{2}\right]$ ), one must optimize over all possible pairings of rotations and landmark-constrained re-parameterizations of $q_{1}$ and $q_{2}$; this process is often referred to as registration or alignment of the two curves. This task can be reduced to fixing $q_{1}$ and searching for the optimal rotation and landmark-constrained re-parameterization pair, $\left(O^{*}, \gamma^{*}\right) \in S O(2) \times \Gamma_{0}$, that optimally aligns $q_{2}$ to $q_{1}$ :

$$
\left(O^{*}, \gamma^{*}\right)=\underset{O \in S O(2), \gamma \in \Gamma_{0}}{\operatorname{argmin}} d_{\mathscr{C}}\left(q_{1}, O\left(q_{2} \circ \gamma\right) \sqrt{\dot{\gamma}}\right)
$$

Finding the optimal rotation is a simple singular value decomposition (SVD) problem, also referred to as Procrustes analysis. In the unconstrained elastic shape analysis framework, the optimal reparameterization was found using a dynamic programming algorithm (Joshi et al., 2007; Srivastava et al., 2011; Kurtek et al., 2012); implementation details are described in Robinson (2012). This approach is not appropriate in the current case due to the hard landmark constraints placed on $\gamma^{*}$. There are two possible approaches to solving the current problem: (1) a gradient descent algorithm over $\Gamma_{0}$, or (2) a product space approach where the optimization over $\Gamma_{0}$ is separated into an optimization over the unconstrained re-parameterization group $\Gamma$ for each segment formed using the landmark constraints. In this paper, we take the second approach due to the computational 


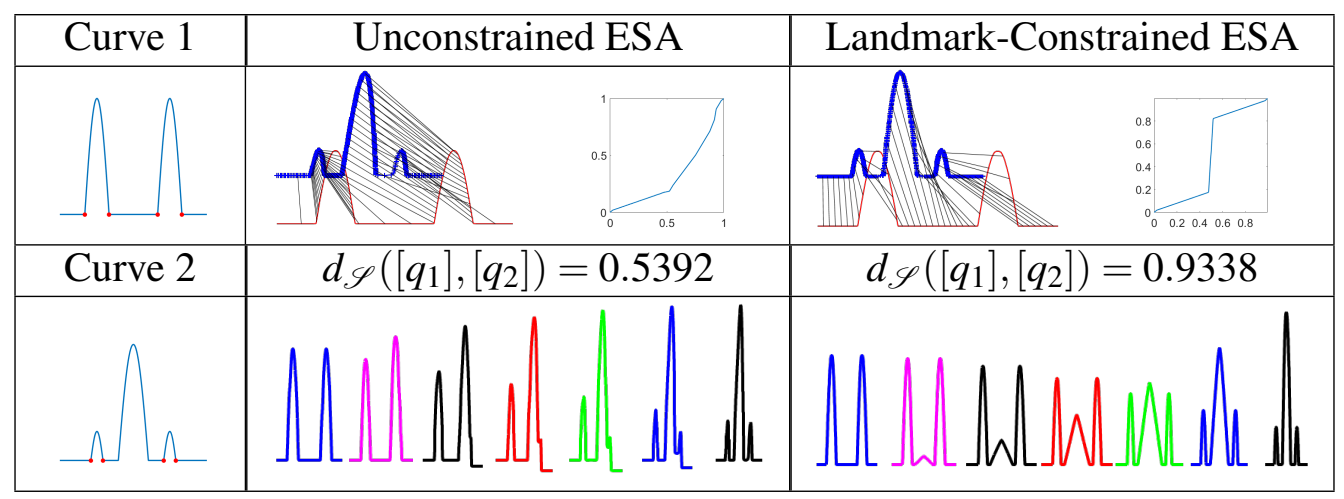

Figure 2: Left: Two landmark-constrained curves. Top: Shape correspondence marked by solid black lines and the optimal re-parameterization $\gamma^{*}$. Bottom: Geodesic paths between the two shapes.

efficiency of the dynamic programming algorithm; we describe the implementation details in the next section. Once the optimal alignment of $q_{2}$ (denoted by $\left.q_{2}^{*}=O^{*}\left(q_{2} \circ \gamma^{*}\right) \sqrt{\dot{\gamma}^{*}}\right)$ is found, the geodesic distance between shapes on $\mathscr{S}$ is given by

$$
d_{\mathscr{S}}\left(\left[q_{1}\right],\left[q_{2}\right]\right)=\cos ^{-1}\left(\left\langle q_{1}, q_{2}^{*}\right\rangle\right)
$$

and the geodesic path is $\alpha_{q_{1}, q_{2}^{*}}$.

Note that the landmark-constrained geodesic distance is always greater than or equal to the unconstrained elastic distance. The distances are equal (and also yield the same geodesic path) if the optimal rotation and re-parameterization are equivalent under the two methods. Thus, if the distances are very different, landmark constraints play a significant role in the shape analysis, and the impact on the geodesic path is striking. This is usually the case when shapes with missing features are being compared, which introduces a high level of ambiguity into the shape registration and comparison. Figure 2 illustrates this phenomenon using a simulated example. We consider two curves which are fairly similar, except the first curve is missing the middle peak. This type 
of situation is very common in many applications, e.g., an occluded limb of an animal or person, or a partially missing anatomical structure due to abnormal pathology. This introduces ambiguity into the re-parameterization process that cannot be easily handled using the unconstrained method. Without including any landmark information, the optimal unconstrained alignment matches the large middle peak on the second curve to the right peak on the first curve; this yields a geodesic path which is unnatural, given what is known about the features of the two curves. However, if landmark information is allowed to 'guide' the elastic shape comparison, then both the correspondence and the geodesic are much more natural. In particular, the missing peak simply grows along the geodesic deformation.

\subsection{Statistics on the Landmark-Constrained Shape Space}

Since $\mathscr{S}$ is a quotient space of a nonlinear manifold, standard vector calculus (like addition, multiplication, etc.) is not applicable. This implies that multivariate statistical techniques require appropriate modification to be applied to the problem at hand. In this section, we provide a recipe for computing statistical summaries of landmark-constrained shapes including the average and covariance. Furthermore, we explore the variability in such shapes using principal component analysis (PCA).

\subsubsection{Mean Shape}

We begin with a procedure to calculate the mean of a collection of shapes. Unfortunately, the mean shape cannot be simply defined by averaging the SRVFs. Instead, we resort to the intrinsic sample

mean on $\mathscr{S}$, called the Karcher mean. For a set of shapes $\left\{\beta_{1}, \ldots, \beta_{n}\right\}$ with corresponding SRVFs 
$\left\{q_{1}, \ldots, q_{n}\right\}$, the sample Karcher mean is defined as:

$$
[\bar{q}]=\underset{[q] \in \mathscr{S}}{\operatorname{argmin}} \sum_{i=1}^{n} d_{\mathscr{S}}\left([q],\left[q_{i}\right]\right)^{2} .
$$

We use one element of this equivalence class as its representative for subsequent statistical analysis. To calculate the Karcher mean we use a gradient descent algorithm (Le, 2001; Kurtek et al., 2013). The Karcher mean algorithm requires the definition of the exponential map on $\mathscr{C}$ and its inverse. These expressions are given analytically due to the well known differential geometry of the unit sphere. Let $T_{q}(\mathscr{C})$ denote the tangent space at a point $q \in \mathscr{C}$. Then, the inverse-exponential map, $\exp ^{-1}: \mathscr{C} \rightarrow T_{q}(\mathscr{C})$ is given by $\exp _{q}^{-1}(\tilde{q})=v=\frac{\theta}{\sin (\theta)}(\tilde{q}-\cos (\theta) q)$, where $\theta$ is the geodesic distance between $\tilde{q}$ and $q$ on $\mathscr{C}$. This map returns the shooting vector $v$, which represents the direction and distance from $q$ to $\tilde{q}$. Since this tangent space is a vector space, standard multivariate statistical methods can be conducted using the shooting vectors. The exponential map $\exp : T_{q}(\mathscr{C}) \rightarrow \mathscr{C}$ maps shooting vectors from the tangent space to the representation space as follows: $\exp _{q}(v)=\tilde{q}=\cos (\|v\|) q+\frac{\sin (\|v\|)}{\|v\|} v$.

\subsubsection{Principal Component Analysis}

Principal component analysis (PCA) is a useful way to visualize the dominant modes of variation in shape data. Unfortunately, since PCA applies to multivariate data on vector spaces, it cannot be performed directly on $\mathscr{S}$; however, tangent PCA can be conducted on the (linear) tangent space using shooting vectors. This process first requires finding the Karcher mean shape $\bar{q} \in[\bar{q}]$. Once this is obtained, for $i=1, \ldots, n$, shooting vectors $v_{i}$ on $T_{\bar{q}}(\mathscr{C})$ are computed via the inverseexponential map. At the implementation stage, the shooting vectors are usually sampled using $N$ 
points, i.e., $v_{i} \in \mathbb{R}^{2 \times N}$. We reshape each $v_{i}$ to a vector in $\mathbb{R}^{2 N}$. The sample covariance matrix is computed using the shooting vectors: $K=\frac{1}{n-1} \sum_{i=1}^{n} v_{i} v_{i}^{T}$. Due to the symmetry of the covariance matrix, its SVD is given by $K=U \Sigma U^{T}$, where $\Sigma$ is a diagonal matrix of principal component variances (arranged in non-decreasing order), and the columns of $U$ are the corresponding modes of variation in the data. One can explore one such direction of variation $U_{i} \in \mathbb{R}^{2 N}$ by computing $v_{t}=t \sqrt{\Sigma_{i i}} U_{i}$ (for some values of $t$ ), where $\Sigma_{i i}$ is the $i$ th diagonal element of $\Sigma$. This vector can be re-shaped back to the original size of $2 \times N$, and mapped to the shape space $\mathscr{S}$ using the exponential map $q_{t}=\exp _{\bar{q}}\left(v_{t}\right)$. The SRVF $q_{t}$ is then converted to $\beta_{t}$ for visualization. As $t$ is varied, one explores the direction of variability in the data specified by the singular vector $U_{i}$.

\subsection{Landmark-Constrained Size-and-Shape Space}

The method described in previous sections removed scale as a feature of comparison by re-scaling all SRVFs to be of unit length. But, if the scale of objects under analysis is deemed important, the framework must be adjusted as follows:

- The pre-size-and-shape space $\mathscr{C}_{s}=\left\{q:[0,1] \rightarrow \mathbb{R}^{2}\right\}$ is now the full $\mathbb{L}^{2}$ space rather than the Hilbert sphere.

- The shape space $\mathscr{S}_{s}$ is defined similarly to the previous case as a quotient space $\mathscr{S}_{s}=$ $\mathscr{C}_{s} /\left(S O(2) \times \Gamma_{0}\right)$

- The geodesic distance between two shapes is given by $d_{\mathscr{S}_{S}}\left(\left[q_{1}\right],\left[q_{2}\right]\right)=\underset{O \in \operatorname{SO}(2), \gamma \in \Gamma_{0}}{\min } \| q_{1}-$ $O\left(q_{2} \circ \gamma\right) \sqrt{\dot{\gamma}} \|$. If $\left(O^{*}, \gamma^{*}\right)$ are the optimal rotation and landmark-constrained re-parameterization, and $q_{2}^{*}=O^{*}\left(q_{2} \circ \gamma^{*}\right) \sqrt{\dot{\gamma}^{*}}$, then the geodesic path between the two shapes in $\mathscr{S}$ is given by the straight line segment $\alpha_{q_{1}, q_{2}^{*}}^{s}(\tau)=(1-\tau) q_{1}+\tau q_{2}^{*}, \tau \in[0,1]$. 
- The tangent space at any point $q \in \mathscr{C}$ can be identified with $\mathbb{L}^{2}$. Thus, the inverse-exponential map becomes $\exp _{q}^{-1}(\tilde{q})=v=\tilde{q}-q$, and the exponential map becomes $\exp _{q}(v)=\tilde{q}=q+v$.

\section{Implementation Details}

We now address some aspects of implementing the landmark-constrained elastic shape analysis method. We begin with the task of optimizing over the rotation and landmark-constrained reparameterization product group. Second, we discuss the issue of curve discretization. Finally, we provide the computational cost for the proposed method under several scenarios. Pseudocode for implementation of the alignment and Karcher mean procedures is provided in the Appendix.

\subsection{Optimization over $S O(2) \times \Gamma_{0}$}

To optimize Equation 1 over $\Gamma_{0}$, one would have to resort to a gradient descent algorithm on that space. While this is a viable approach, it is computationally expensive. Thus, we solve this problem by dividing it into several optimization problems over the unconstrained re-parameterization group $\Gamma$, which can be solved very efficiently using the dynamic programming algorithm (Bertsekas, 1995). We provide the details of our approach next.

Consider two open curves $\beta_{1}$ and $\beta_{2}$ (this approach works for closed curves with minor adjustments). Assume that each of the curves has the same number of $k$ landmarks, not counting the start and end points of the curves. Then, each curve can be split into a set of $k+1$ separate curve segments that are in correspondence: $\left\{\beta_{1}^{1}, \ldots, \beta_{1}^{k+1}\right\}$ and $\left\{\beta_{2}^{1}, \ldots, \beta_{2}^{k+1}\right\}$. In other words, curve segments $\beta_{1}^{i}$ and $\beta_{2}^{i}$ for $i=1, \ldots, k+1$ are matched. Each of these segments is also an open, absolutely continuous curve, which can be represented using its SRVF. Let $q_{1}$ and $q_{2}$ be the SRVFs for 
$\beta_{1}$ and $\beta_{2}$, and $\left\{q_{1}^{1}, \ldots, q_{1}^{k+1}\right\}$ and $\left\{q_{2}^{1}, \ldots, q_{2}^{k+1}\right\}$ be the SRVFs for the associated curve segments.

In the unconstrained framework, rotation and re-parameterization alignment was performed globally, i.e., the optimal transformations were found for the entire curve. Similarly, a first proposition may be to find the optimal rotation and re-parameterization for each curve segment separately, and then 'glue' the results together to obtain the full shape. However, such an approach does not preserve the global shape of the object under analysis. The method taken in McCane (2012) fixes this by finding the re-parameterization for each segment using a local rotation; once the optimal re-parameterization is found, the local rotations are discarded and an optimal rotation is found for the entire curve. This does preserve the shapes of the objects, but the optimal re-parameterization is dependent on the local rotations, which are unused in subsequent analyses. Instead, optimization over the rotation group $S O(2)$ should be considered globally (for the whole curve) rather than locally (for each curve segment), for all steps of the alignment process. Applying different reparameterizations to each curve segment does not alter the appearance of the global shape. Thus, the optimization over $\Gamma_{0}$ will be achieved by finding local re-parameterizations in $\Gamma$ for each curve segment.

Optimization over $S O(2)$ is performed using Procrustes analysis. First, we compute $A=$ $\int_{0}^{1} q_{1}(t) q_{2}(t)^{T} d t$. We find the SVD of $A$ as $A=U \Sigma V$. The optimal rotation is given by $O^{*}=U V^{T}$ (last column of $V$ changes sign in case $\operatorname{det}(A)=-1$ ). To find the optimal landmark-constrained reparameterization, we first optimize over the product space $\Gamma_{k+1}=\Gamma \times \cdots \times \Gamma$. This optimization is performed segment-wise using the dynamic programming algorithm resulting in $\gamma_{1}^{*}, \ldots, \gamma_{k+1}^{*}$. The global landmark-constrained re-parameterization $\gamma^{*}$ is obtained by concatenating the $\gamma_{i}^{*}$ s. Note that during this step, the interval on which each $\gamma_{i}^{*}$ is defined must be re-scaled proportionally to the length of the curve segment $\beta_{2}^{i}$. In order to optimize over the product group $S O(2) \times \Gamma_{0}$, we 
iterate between individual optimizations over $S O(2)$ and $\Gamma_{0}$ until convergence.

\subsection{Discretization}

Since the proposed functional representations are infinite-dimensional, curve discretization (in the form of a finite set of points) is necessary in order to implement the proposed method. We allow the specification of landmarks in two different ways: (1) by manually selecting points of interest on the given curves and splitting the curves into corresponding segments, and (2) by directly providing curve segments obtained using some other automatic method. Once the segments are obtained, we resample each segment, using arc-length, to 100 points via linear interpolation. We have found through multiple examples that this sampling is fine enough to capture very intricate details of the curves under study (see examples in Figures 4 and 5). We use finite differences (forward and backward for extremal points and central for interior segment points) to approximate all derivatives and the trapezoidal rule to approximate all integrals. A general sense of the continuous geodesic path between shapes can be obtained by sampling seven equally spaced points along the path (the first and last of which correspond to the two original shapes being compared).

\subsection{Computational Cost}

All computation using the landmark-constrained elastic shape analysis method is performed in MATLAB, version R2015b (except the dynamic programming algorithm which was implemented in C), on an ASUS F555UA-EH71 personal laptop with 8 GB of RAM and an Intel Core i7 processor. The time needed to compute the geodesic distance and path between two shapes is dependent on the number of specified landmarks and points sampled on each curve segment. We examine the 


\begin{tabular}{|c|c|c|c|c|c|c|c|c|c|c|}
\hline No. of Landmarks & 1 & 2 & 3 & 4 & 5 & 6 & 7 & 8 & 9 & 10 \\
\hline Avg. Duration & 0.0272 & 0.0470 & 0.0680 & 0.0886 & 0.1072 & 0.1277 & 0.1458 & 0.1656 & 0.1869 & 0.2066 \\
\hline
\end{tabular}

Table 1: Average duration (seconds) of 1000 alignments with a fixed number of landmarks.

impact of the number of landmarks on the computational cost using two shapes from the MPEG07 dataset (described in the subsequent section). For each number of specified landmarks, we report the average elapsed time to align the two curves (optimize over $S O(2) \times \Gamma_{0}$ ) over 1000 trials. This result is given in Table 1. Clearly, an increase in the number of landmarks leads to a higher computational cost; however, none of the reported times are unreasonable. Each landmark appears to contribute approximately 0.02 seconds to the constrained alignment process. The sampling density of each segment in this example was fixed to 100 points.

Karcher mean computation is much lengthier for two main reasons: (1) the convergence of the gradient descent algorithm can take many iterations, and (2) the alignment of every shape in the sample to the current "average" is required at each iteration. To explore the computational cost of the algorithm, we use a sample of 30 mice from the mice $\mathrm{T} 2$ vertebrae dataset (described in the subsequent section). Each vertebra had six landmarks and each resulting curve segment was sampled with 100 points. The computation of the Karcher mean required 249 iterations and a total of 716.3423 seconds (2.8769 seconds per iteration on average). We note that the computational cost is highly dependent on the choice of the step size and stopping criterion, which must be specified in the algorithm. As a second example, we computed the Karcher mean of 15 fly wings from the Hawaiian Drosophila dataset (described in the subsequent section). Each fly wing has a total of nine landmarks and each segment was again sampled using 100 points. Here, the Karcher mean algorithm required 74 iterations to converge and a total of 294.9959 seconds (3.9864 seconds per iteration on average). 


\section{Practical Applications}

In this section, we showcase the proposed landmark-constrained elastic shape analysis (LC ESA) framework using three applications. First, we provide comparisons of landmark-constrained geodesic deformations to the unconstrained elastic shape analysis (UC ESA) case. For one example, we also compare the proposed method to bending only methods (arc-length parameterization and semilandmarks). This set of results is reported on the well-known MPEG-7 dataset ${ }^{1}$. The second application studied in this paper is the landmark-constrained elastic size-and-shape analysis of the second thoracic (T2) mice vertebrae. This dataset, available in R as part of the 'shapes' package ${ }^{2}$, contains T2 vertebrae for three groups (30 control, 23 large and 23 small) (see Dryden and Mardia (1998); Cheng et al. (2016) for more details). The large and small groups consist of mice that were genetically selected for large and small body weights; the control group were those that were not genetically selected. Each vertebra is available as a set of six landmarks based on points of extreme curvature (mathematical rather than anatomical landmarks) with additional nine semi-landmarks placed between each pair of landmark points (for a total of 60 points). Thus, this dataset allows for the statistical analysis to be performed using landmarks only (landmark-based shape analysis), outlines only (UC ESA), or landmarks and outlines jointly (LC ESA). Figure 3(a) displays an example mouse vertebra with labeled landmarks and anatomical structures of interest. The final application considers comparisons and clustering of Hawaiian Drosophila fly wings. The data was reported in Edwards et al. (2007) and later analyzed in McCane (2012). Each wing is composed of an outline and major longitudinal veins II-V. There is a total of nine landmarks on each wing representing intersections of major structures. Figure 3(b) displays an example fly wing with labeled landmarks

\footnotetext{
${ }^{1}$ http://www.dabi.temple.edu/ shape/MPEG7/dataset.html

${ }^{2}$ https://cran.r-project.org/web/packages/shapes/index.html
} 


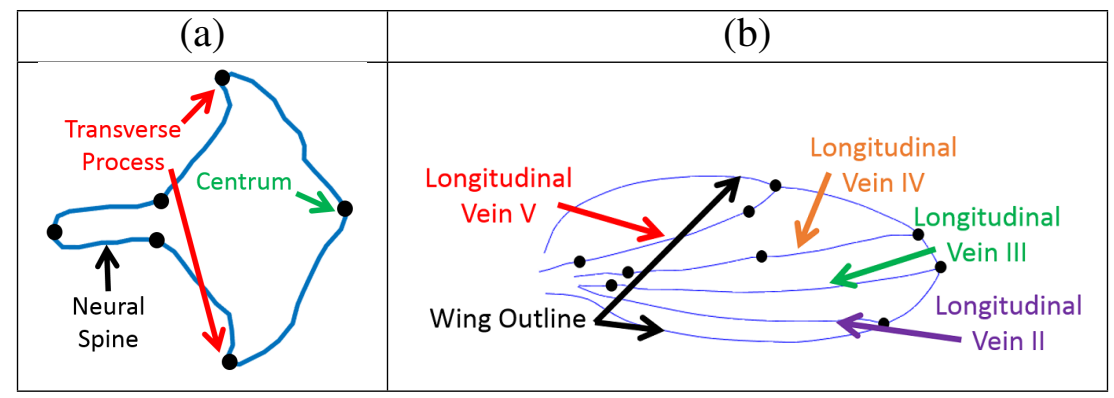

Figure 3: (a) Anatomy of a mouse vertebra. (b) Anatomy of a Hawaiian Drosophila fly wing. All landmarks are labeled in black.

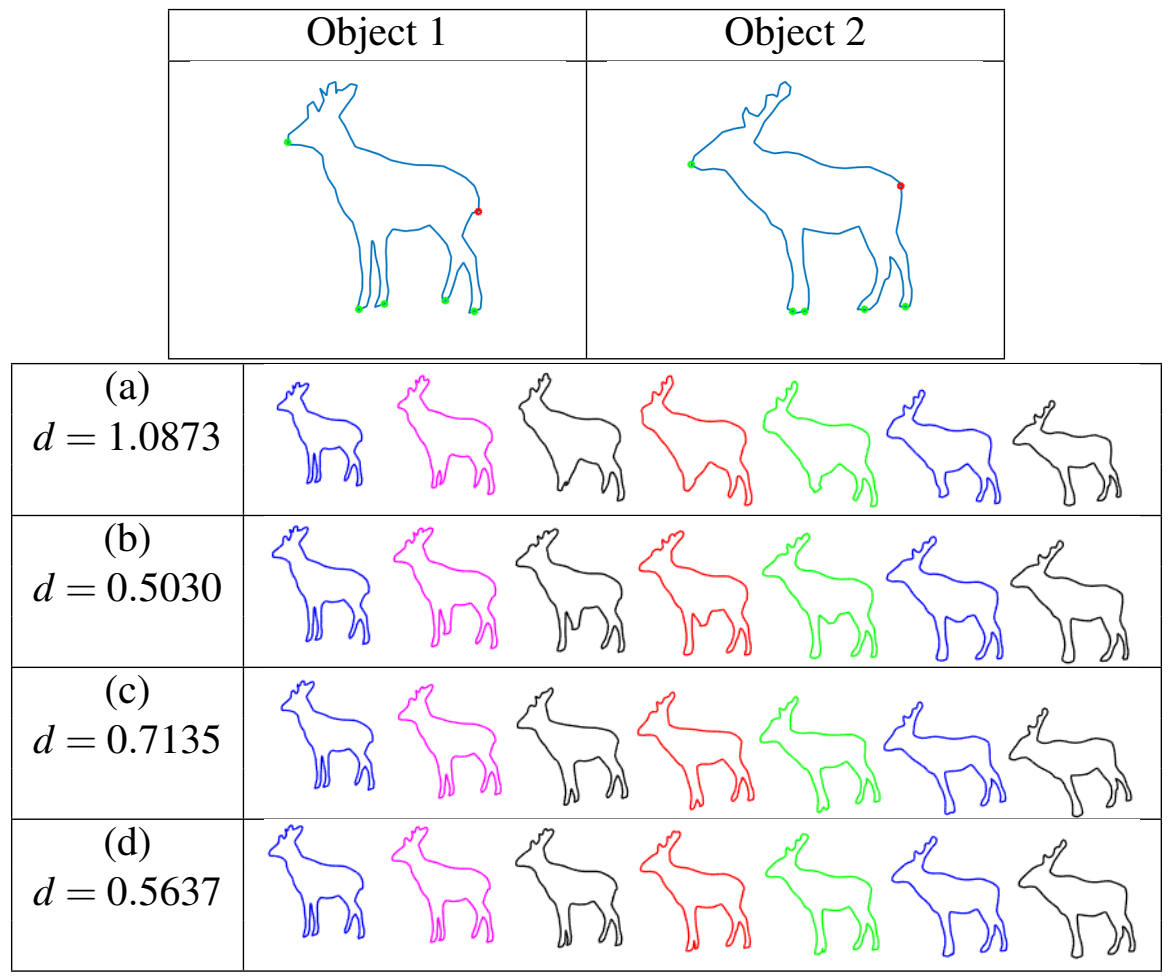

Figure 4: Motivating example from the MPEG-7 dataset. Top: Two different deer outlines annotated with semantic landmarks (green). The starting point is marked in red. Bottom: Comparison of geodesic paths and distances for (a) arc-length parameterization, (b) UC ESA, (c) semi-landmarks, and (d) LC ESA.

and anatomical structures of interest. 


\subsection{Landmark-Constrained Geodesic Deformations of MPEG-7 Data}

We begin with several geodesic comparisons between the UC ESA and proposed LC ESA frameworks. To do this, we selected pairs of complex objects from the MPEG-7 dataset. We begin with a motivating example where the benefits of LC ESA are very clear. This result is presented in Figure 4. The two objects under study are deer outlines, which are very different in structure. Perhaps the most evident difference is that one of the legs is occluded by the other in the second deer outline. We compare the geodesic path and distance between the two outlines for four different methods. The first two are unconstrained methods: (a) uses an arc-length parameterization over the unconstrained outline while (b) uses UC ESA. The last two are constrained methods, with (c) using equally spaced semi-landmarks between the marked landmark points, and (d) using the proposed LC ESA method. Notice that going from the arc-length parameterization based method to UC ESA yields a drastic improvement in the geodesic path. This is due to improved matching of geometric features across the two curves, which is achieved by finding the optimal re-parameterization (rather than assuming arc-length). This also greatly reduces the geodesic distance between the two shapes. However, one major problem for the UC ESA method is that it cannot determine how to deal with the occluded feature. As a result, the missing leg grows out of the stomach along the geodesic path, which is a very unnatural deformation between the two shapes. Note that the geodesic distance measures the magnitude of this deformation and inherits the same disadvantage.

To improve this result, both deer can be annotated with a few (six) landmarks marking semantic features on both objects. The most important landmark corresponds to the missing leg, which can indicate that the leg is occluded. The resulting semi-landmark and LC ESA geodesics are much more natural, where the single leg splits into two, an effect that appears as if the deer moved one 
of his legs. This deformation is accompanied by a larger, yet more natural, geodesic distance (as compared to the UC ESA method). However, while the semi-landmark method also improves upon the UC ESA geodesic, parts of the deformation are less natural than the deformation based on the LC ESA approach. This is especially seen in the antlers (pronounced geometric features are not preserved) and the occluded leg (a wide gap between the legs is introduced along the geodesic). This approach inherits drawbacks of the arc-length parameterization approach, as the semi-landmarks are equally spaced between landmarks. As a result, if a segment between two landmarks has many fine details, the LC ESA method shows major improvements over the semilandmark method.

We present more comparisons of geodesics computed under the UC ESA and LC ESA methods in Figure 5, where we observe very similar results (especially for the dog and stingray examples). When the addition of landmarks does not affect the resulting geodesic, this is also reflected in the geodesic distances as expected (crown example).

\subsection{Landmark-Constrained Elastic Shape Analysis of Mice Vertebrae}

Mean shapes were calculated for the T2 mice vertebrae data separately for each group. Scale is included in this analysis as a feature of note; thus, all statistics were computed on the landmarkconstrained size-and-shape space $\mathscr{S}_{s}$. The average mice vertebrae are displayed in Figure 6(a), along with plots of the Karcher variance at each iteration in panel (b). The Karcher variance plots indicate that the algorithm converged for all groups. Lengths of the averages were also calculated and are reported below the respective plots. First, note that there are clear differences in the shape of the neural spine and centrum regions of the vertebrae. It is also interesting to note that the length 


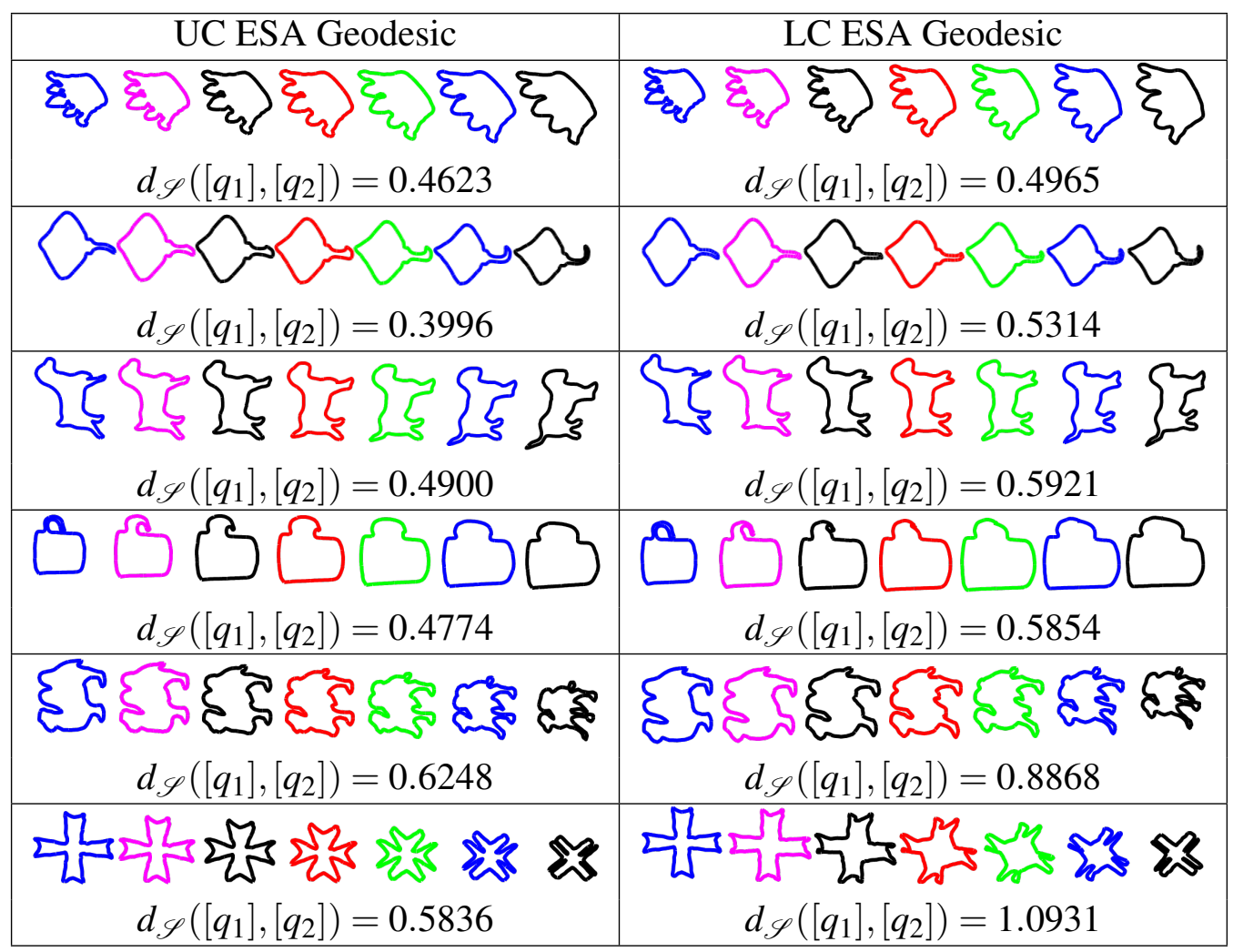

Figure 5: Comparison of additional geodesics for various shapes.

\begin{tabular}{|c|c|c|c|}
\hline Group & Control & Large & Small \\
\hline (a) & & & \\
\hline & 1000 & 我地 & \\
\hline & $\infty \infty$ & $\infty$ & sol \\
\hline & 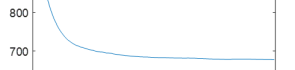 & sol & \\
\hline (b) & 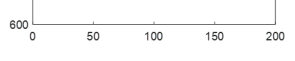 & 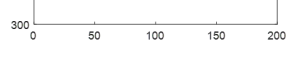 & 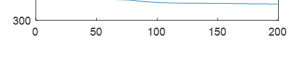 \\
\hline (c) & Length $=565.7$ & Length $=585.8$ & Length $=504.9$ \\
\hline
\end{tabular}

Figure 6: (a) Landmark-constrained average vertebrae shapes for the three mouse groups in the dataset; sizes have been re-scaled for improved display. (b) Convergence plots for the Karcher mean algorithm with the number of iterations on the $x$-axis and the Karcher variance on the $y$-axis. (c) Length of the Karcher mean for each group. 


Group

Figure 7: Three principal directions (PD) of variability in the T2 vertebrae in mice for the three groups. Blue $=-3$ standard deviations (sd); Green $=-2$ sd; Red $=-1$ sd; Magenta=Karcher mean; Black $=+1 \mathrm{sd} ;$ Cyan=+2 sd; Brown=+3 sd. The bottom row displays the length of the mice vertebrae ( $y$-axis) corresponding to -3 to +3 standard deviations ( $x$-axis) for the three principal directions. Blue=PD1; Red=PD2; Yellow=PD3.

\begin{tabular}{|c|c|}
\hline & KV \\
\hline \hline Control & $1.394 \times 10^{4}$ \\
Large & $1.013 \times 10^{4}$ \\
Small & $8.696 \times 10^{3}$ \\
\hline
\end{tabular}

Table 2: Karcher variance (KV) for each group in the mice vertebrae data.

of the control Karcher mean is much closer to the large group than the small group.

As a next step, we performed group-wise tangent PCA. Figure 7 shows the results for the three 


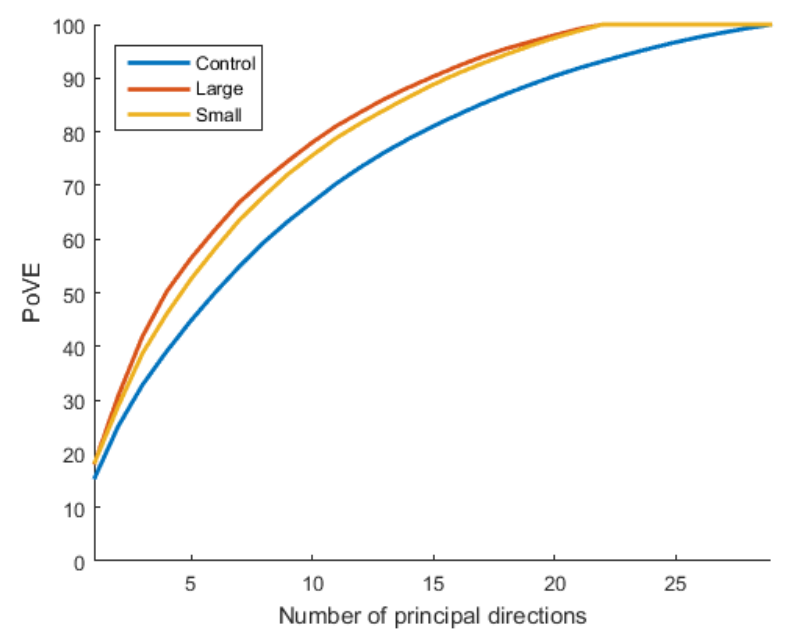

Figure 8: Percentage of variation explained (PoVE) vs. number of principal directions for each group in the mice vertebrae data.

principal directions of variation for each group in the given data, using integer values of $t$ from -3 to 3 , i.e., each direction is displayed from -3 standard deviations to +3 standard deviations with $t=0$ corresponding to the Karcher mean. The dominant mode of variability in the small group reflects significant shape changes in the neural spine region of the vertebrae. The second and third principal directions of variation have little variance. The third direction appears to be capturing shape changes in the transverse process. All three of the principal modes of variability in the large group capture significant shape changes in the neural spine. The principal direction also captures a shape change in the centrum. The control group appears to not have quite as much shape variability as the other two groups. The size variability is displayed in the bottom of Figure 7. The length of the mice vertebrae corresponding to -3 to +3 standard deviations for the top three modes of variability is plotted. The large group appears to have significant size changes in the first and second principal directions of variability. The small group captures the main size change in the first principal direction. Again, surprisingly, the control group does not vary much in size in the three principal directions. 


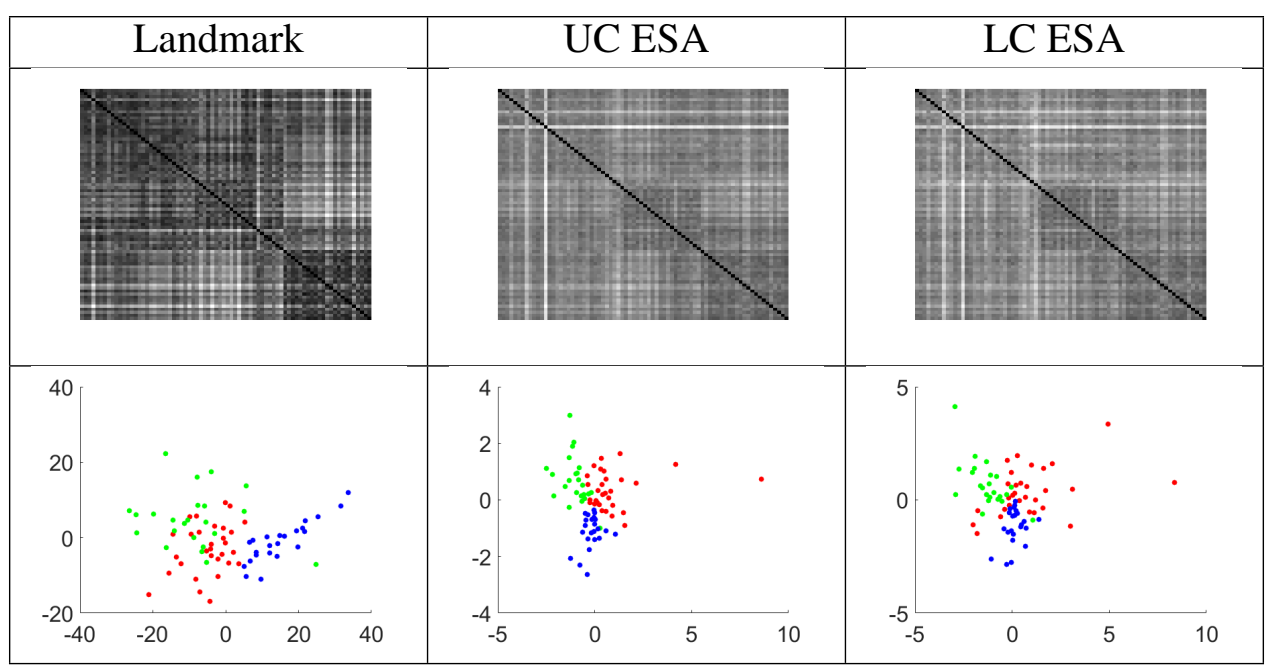

Figure 9: Top: Image of pairwise distance matrix. Bottom: Two-dimensional MDS plots with control in red, small in blue and large in green.

As in other statistical applications, PCA is a useful tool for dimension reduction. Figure 8 shows the amount of variation explained as a function of the number of principal components for each group. Both the large and small groups had smaller sample sizes $(n=23$ for these two groups) than the control group. The control group lags behind the other two groups in terms of the proportion of variation accounted for by each principal direction of variability. This could be due to the larger sample size in this group and increased variability due to size of the vertebrae. The total variance in each group is also reported in Table 2. The small group has markedly smaller variance than the other two.

\subsubsection{Classification Performance}

One way to assess the performance of shape analysis is to study classification of shapes into established groups. In this application, there is a need to automatically classify vertebrae from individual mice using their size-and-shape as there is little or no other information available (Cheng et al., 2016). Furthermore, this dataset lends itself to a comparison of the landmark-based, fully elastic 


\begin{tabular}{|c|c|c|c|}
\hline & Landmark & UC ESA & LC ESA \\
\hline \hline Control & 5 & 5 & $\mathbf{2}$ \\
\hline Large & 5 & $\mathbf{2}$ & 3 \\
\hline Small & 2 & 2 & $\mathbf{1}$ \\
\hline \hline Total & 12 & 9 & $\mathbf{6}$ \\
\hline
\end{tabular}

Table 3: LOO-NA misclassification number for the three mice groups based on the three shape analysis approaches. Best performance is highlighted in bold.

and landmark-constrained elastic methods. For each method, we calculated the distance matrices, where the three clustered groups are somewhat evident as blocks (arranged in the same order as in the data description) in the plots in Figure 9. To make the clusters more apparent, we also include two-dimensional plots using the multidimensional scaling (MDS) method. The clusters appear well separated using the UC ESA and LC ESA methods. The landmark-based method performs worst in this case.

In order to classify the vertebrae, we consider a leave-one-out nearest average (LOO-NA) approach for each method. For each vertebra, average shapes are computed for the three groups in a leave-one-out manner. Then, geodesic distances are calculated between the current vertebra and the three averages; the vertebra is classified into the group corresponding to the smallest distance. Table 3 displays the number of misclassified observations per group and method. Overall, the LC ESA method provides best performance. In particular, it provides smallest misclassification rates for the control and small groups, and a slightly higher misclassification rate than UC ESA for the large group. Thus, in this application, the proposed approach provides improvement over competing methods. Further improvement in classification could result from the consideration of landmark constraints as soft, rather than hard. This would allow for landmark points to shift slightly during analysis in order to reflect uncertainty in the landmark placement. We leave such 
analysis as future work.

\subsubsection{Two-Sample Hypothesis Tests}

Formal statistical inference can additionally be conducted using the LC ESA method. In many applications, there may be interest in comparing groups according to mean shape. That is, if $\left[\mu_{1}\right]$ and $\left[\mu_{2}\right]$ are the population mean shapes for two groups, we would like to perform the following two-sample hypothesis test: $H_{0}:\left[\mu_{1}\right]=\left[\mu_{2}\right]$ versus $H_{1}:\left[\mu_{1}\right] \neq\left[\mu_{2}\right]$. A common approach in conducting this test without making distributional assumptions is to perform a permutation test (as shown in Good (1994) and Dryden and Mardia (1998) for landmark shape analysis). To do this, we

first find $d_{0}=d_{\mathscr{S}}\left(\left[\bar{q}_{1}\right],\left[\bar{q}_{2}\right]\right)$, the shape distance between the (SRVFs of the) sample Karcher means of the two groups. Note that this distance can also be computed on the size-and-shape space $\mathscr{S}_{s}$ if size is of interest. Once this is done, for $b=1, \ldots, B$, we randomly permute the group labels of all shapes. For each permutation $b$, the sample Karcher averages are calculated for the two groups, and the shape distance between them is computed. The $B$ different distances form an approximate distribution for the distance between the averages under the null hypothesis; then, if the observed distance $d_{0}$ lies in the tail of this distribution we reject $H_{0}$. The approximate p-value is computed using standard permutation test procedures by observing the fraction of distances under random label permutation equal to or greater than $d_{0}$.

We perform three pairwise two-sample hypothesis tests for differences in average size-andshape of the mice vertebrae: control vs. large, control vs. small, and small vs. large. For each test, we chose $B=100$ and performed a permutation test using the procedure described above. Note that distances were calculated on $\mathscr{S}_{S}$ since the size of the vertebrae plays an important role in this application. Figure 10 displays the generated histograms of the test statistic under $H_{0}$ for 


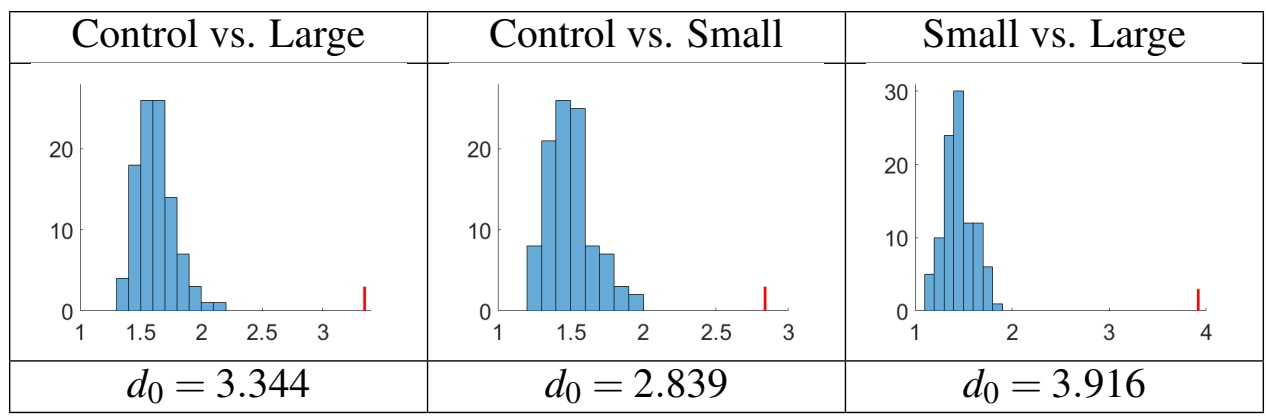

Figure 10: Histograms of distances from the three pairwise two-sample permutation tests. The red vertical line indicates the value of the test statistic $d_{0}$ computed using the correct group labels.

each of the three hypothesis tests; the vertical red line shows the value of the test statistic $d_{0}$ computed using the correct labels. It is clear that the observed distance lies in the tail in each case (p-value $<0.01$ ). Using the standard 0.05 significance level, there is strong evidence that there is a difference in size-and-shape means for the control and large groups, the control and small groups, and the small and large groups.

\subsection{Landmark-Constrained Elastic Shape Analysis of Hawaiian Drosophila}

\section{Fly Wings}

Due to the complex structure of the Hawaiian Drosophila fly wings we must make small adjustments to the presented method to analyze their size-and-shape. We note that the wing object cannot be represented using a single parameterized curve, and thus the UC ESA framework does not apply here. Thus, we use the given landmarks to split the wing into a set of open segments. The full (SRVF) representation space is now a product of $\mathbb{L}^{2}$ spaces. The optimal rotation is again found globally by concatenating the SRVFs of the individual open segments and then solving the Procrustes problem. The optimal re-parameterization is found individually for each pair of segments. It does not make sense to combine the local re-parameterizations into a global one in this 


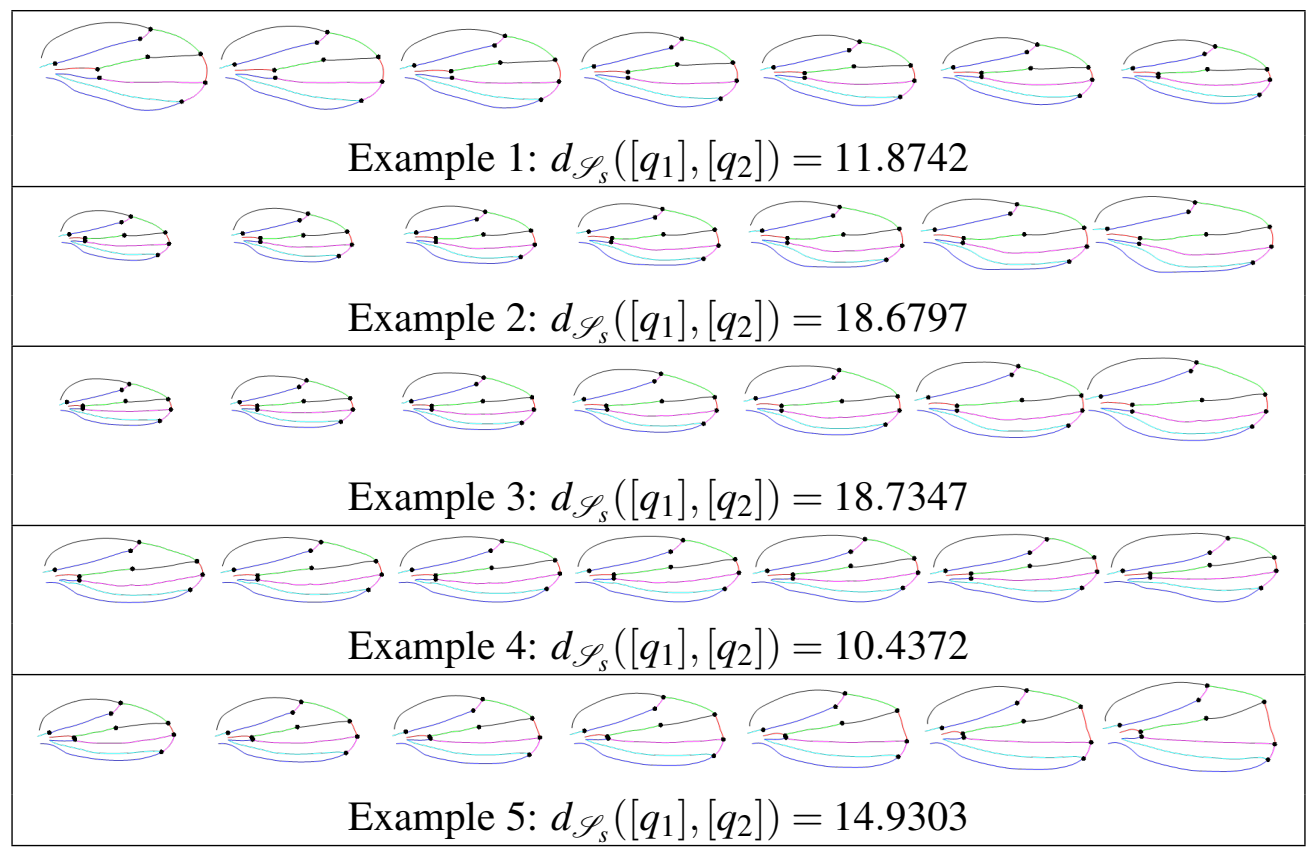

Figure 11: Five examples of size-and-shape geodesic paths and distances between Drosophila fly wings. Corresponding segments are marked with the same color. We highlight the landmark points used in this analysis.

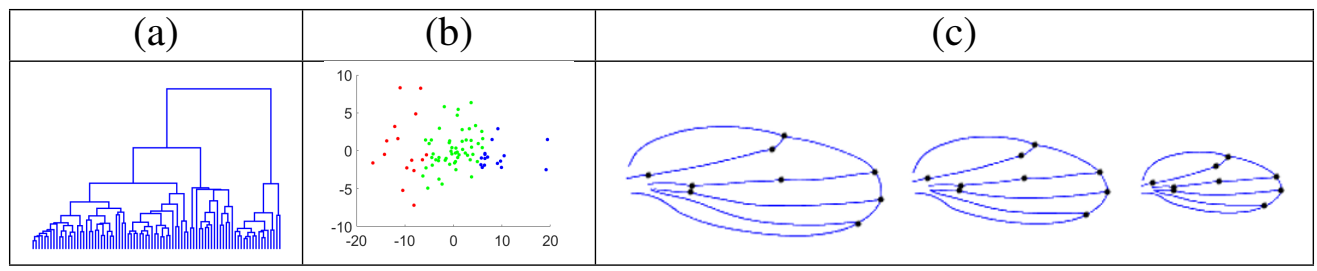

Figure 12: (a) Dendrogram for fly wing data. (b) Two-dimensional MDS plot showing three distinct clusters. (c) The Karcher mean for each cluster of fly wings.

application. Finally, the geodesic distance and path are found using the product space structure.

We begin by presenting several geodesics between different Drosophila fly wings in Figure 11. In Examples 2 and 3, we notice a difference in wing size as well as shape differences between the wing outlines and the longitudinal vein (LV) II. In Example 5, we notice a drastic difference in the length and shape of the segment along the wing outline connecting LVs III and IV. In general, the computed size-and-shape geodesics represent very natural morphological changes of Drosophila fly wings. 


\begin{tabular}{|c|cc|cc|cc|}
\hline & \multicolumn{2}{|c|}{ Cluster 1 } & \multicolumn{2}{c|}{ Cluster 2 } & \multicolumn{2}{c|}{ Cluster 3 } \\
\hline Characteristic & Avg. & St. Dev. & Avg. & St. Dev. & Avg. & St. Dev. \\
\hline \hline Length & 913.1 & 66.9 & 701.4 & 82.0 & 507.3 & 67.5 \\
\hline Width & 382.4 & 59.6 & 303.5 & 35.9 & 215.9 & 28.6 \\
\hline Length/Width & 2.429 & 0.313 & 2.319 & 0.198 & 2.352 & 0.126 \\
\hline
\end{tabular}

Table 4: Summary statistics (average and standard deviation) of global shape characteristics for the three discovered clusters.

To discover size-and-shape patterns in the given data, we performed hierarchical clustering with a 'complete' linkage function. We discovered three main clusters as shown in the dendrogram and MDS plots in Figure 12(a) and (b). Figure 12(c) shows the Karcher average fly wing in each cluster. It appears that the main difference between the discovered clusters is the size of the wings. To check whether we are only finding size differences, we computed the average and standard deviation of three characteristics of the global fly wing shape in each cluster: (1) length, (2) width and (3) the ratio of length to width. It appears that the lengths and widths of fly wings follow the following trend: cluster $1>$ cluster $2>$ cluster 3 . But, when we look at the ratio of length to width we notice something interesting. The fly wings in cluster 3 (group with smallest wings) have a higher average ratio than those in cluster 2 (group with medium wings). This suggests not only a size difference between the clusters, but also a general shape difference. A better method to visualize differences between the discovered clusters would be to use PCA as was done for the mice vertebrae. Tangent PCA could be done for each of the wing segments individually; however, this does not account for variability induced by the dependence between the different fly wing segments (which are joined together to obtain the full fly wing). Thus, it is difficult to capture this dependence while simultaneously visualizing the variability in clusters. 


\section{Summary and Future Research}

This paper introduces a novel statistical framework for elastic shape analysis of landmark-constrained, planar curves. This method proves useful when, in addition to the object outline, a set of prespecified landmarks (mathematical and/or anatomical) is known, and when object outlines under study include prominent missing features. The key premise of this work is to define a landmarkconstrained group of re-parameterizations and to find correspondences between shapes by optimizing over this group. This is performed by breaking up the object outline of interest according to landmark locations. We provide tools for computation of geodesic paths and distances for shape comparisons, and shape averaging and summarization of variability in different shape classes. We demonstrate the proposed methods using various complex examples from the MPEG-7 dataset as well as real applications to landmark-constrained size-and-shape analysis of mouse vertebrae and Hawaiian Drosophila fly wings.

An interesting and important application of the proposed method is in the comparison and statistical modeling of objects, which are missing important features. Such examples often arise in computational anatomy where biological structures may be missing important parts due to abnormal pathologies. While we have not considered this application in the current paper, we plan to study the performance of the proposed method in such a setting in the future.

We have identified three other major future directions for this work. First, we will develop statistical models for automated detection of landmark points, which would then allow fully automated, landmark-constrained shape analysis. While automatic landmark detection may not work well for all shapes (especially objects with missing geometric features), it would eliminate the manual annotation process for simpler shapes. Second, we will consider the case of soft landmark 
constraints, which additionally reflect uncertainty in landmark placement. This can be approached from the Bayesian perspective with appropriate landmark-enforcing priors placed on the group of re-parameterizations. Lastly, we will try to derive the distribution for the geodesic distance test statistic for the two-sample hypothesis test. This would allow us to circumvent the use of a permutation test, which is computationally expensive.

\section{Acknowledgments}

We thank the associate editor and reviewers for providing valuable feedback. This research has been supported by the NSF under grant number DMS-1209194 (thanks to Steven MacEachern). The views in this paper are not necessarily those of the NSF. 


\section{References}

Bertsekas, D. P. (1995). Dynamic Programming and Optimal Control. Athena Scientific.

Bookstein, F. L. (1986). Size and shape spaces for landmark data in two dimensions. Statistical Science 1(2), 181-222.

Cheng, W., I. L. Dryden, and X. Huang (2016). Bayesian registration of functions and curves. Bayesian Analysis 11(2), 447-475.

Dryden, I. L. and K. V. Mardia (1992). Size and shape analysis of landmark data. Biometrika 79(1), 57-68.

Dryden, I. L. and K. V. Mardia (1998). Statistical Shape Analysis. New York: Wiley.

Edwards, K. A., L. T. Doescher, K. Y. Kaneshiro, and D. Yamamoto (2007). A database of wing diversity in the Hawaiian Drosophila. PLOS ONE 2(5), e487+.

Good, P. (1994). Permutation Tests. New York: Springer-Verlag.

Joshi, S. C. and M. I. Miller (2000). Landmark matching via large deformation diffeomorphisms. IEEE Transactions on Image Processing 9(8), 1357-1370.

Joshi, S. H., E. Klassen, A. Srivastava, and I. H. Jermyn (2007). A novel representation for Riemannian analysis of elastic curves in $\mathbb{R}^{n}$. In Proceedings of the IEEE Conference on Computer Vision and Pattern Recognition, pp. 1-7.

Kendall, D. G. (1984). Shape manifolds, Procrustean metrics, and complex projective shapes. Bulletin of London Mathematical Society 16, 81-121.

Klassen, E., A. Srivastava, W. Mio, and S. H. Joshi (2004). Analysis of planar shapes using geodesic paths on shape spaces. IEEE Transactions on Pattern Analysis and Machine Intelligence 26(3), 372-383.

Kurtek, S., A. Srivastava, E. Klassen, and Z. Ding (2012). Statistical modeling of curves using shapes and related features. Journal of the American Statistical Association 107(499), 11521165.

Kurtek, S., J. Su, C. Grimm, M. Vaughan, R. T. Sowell, and A. Srivastava (2013). Statistical analysis of manual segmentations of structures in medical images. Computer Vision and Image Understanding 117(9), 1036-1050.

Le, H. (2001). Locating Frechet means with application to shape spaces. Advances in Applied Probability 33(2), 324-338.

Liu, W., A. Srivastava, and J. Zhang (2010). Protein structure alignment using elastic shape analysis. In Proceedings of the First ACM International Conference on Bioinformatics and Computational Biology, pp. 62-70. 
Marron, J. S., J. O. Ramsay, L. M. Sangalli, and A. Srivastava (2015). Functional data analysis of amplitude and phase variation. Statistical Science 30(4), 464-484.

McCane, B. (2012). Shape variation in outline shapes. Systematic Biology 62(1), 134-146.

Robinson, D. T. (2012). Functional Data Analysis and Partial Shape Matching in the Square Root Velocity Framework. Ph. D. thesis, Florida State University.

Small, C. G. (1996). The Statistical Theory of Shape. Springer.

Soatto, S. and A. J. Yezzi (2002). DEFORMOTION: Deforming motion, shape average, and the joint registration and segmentation of images. In Proceedings of the European Conference on Computer Vision, pp. 32-47.

Srivastava, A., E. Klassen, S. H. Joshi, and I. H. Jermyn (2011). Shape analysis of elastic curves in Euclidean spaces. IEEE Transactions on Pattern Analysis and Machine Intelligence 33, 14151428.

Wang, S., J. X. Ji, and Z. Liang (2003). Landmark-based shape deformation with topologypreserving constraints. In Proceedings of the IEEE International Conference on Computer Vision, pp. 923-930.

Younes, L. (1998). Computable elastic distance between shapes. SIAM Journal of Applied Mathematics 58(2), 565-586.

Zahn, C. T. and R. Z. Roskies (1972). Fourier descriptors for plane closed curves. IEEE Transactions on Computers 21(3), 269-281. 